\title{
Vesicle coating and uncoating: controlling the formation of large COPII-coated carriers
}

\author{
Anna K Townley and David J Stephens*
}

\author{
Address: Cell Biology Laboratories, Department of Biochemistry, University of Bristol, School of Medical Sciences, University Walk, \\ Bristol, BS8 1TD, UK \\ * Corresponding author: David J Stephens (david.stephens@bristol.ac.uk) \\ Fl000 Biology Reports 2009, I:65 (doi:I0.34I0/BI-65)
}

The electronic version of this article is the complete one and can be found at: http://F/000.com/Reports/Biology/content/I/65

\begin{abstract}
The basic mechanisms underlying the formation of coated vesicles are now defined in considerable detail. This article highlights recent developments in our understanding of the problem of exporting large macromolecular cargo such as procollagen from the endoplasmic reticulum and discusses the implications that this has for cell and tissue organisation and human disease.
\end{abstract}

\section{Introduction and context}

Transport of molecules between intracellular membranebound compartments is vital for organelle homeostasis and secretion. Genetic and biochemical studies provided the basis for the vesicular transport hypothesis, which begins with protein sorting, vesicle budding, vesicle targeting, and finally vesicle fusion [1]. The first membrane-trafficking step in the secretory pathway, the export of secretory cargo from the endoplasmic reticulum (ER), requires the coat protein II (COPII) coat complex [2]. COPII is a multi-protein complex that is built up sequentially through Sar1-dependent recruitment of other cytosolic components to the membrane. In metazoans (and the yeast Pichia pastoris but notably not Saccharomyces cerevisiae [3]), COPII assembly occurs at specialised zones of ER membrane that are defined by electron microscopy (EM) and called transitional ER $[2,4]$. Together with the nascent budding structures, the transitional ER and other COPII-labelled profiles go to make up an ER exit site (ERES), a term originating largely from light microscopy observations. The small guanine triphosphate (GTP)-binding protein Sar1 is recruited to the ER membrane following guanine diphosphate (GDP)/GTP exchange by its guanine nucleotide exchange factor (GEF), Sec12. Sar1-GTP is stabilised on the membrane via insertion of an $\mathrm{N}$-terminal alpha helix into the lipid bilayer; this can drive deformation of the membrane and even fission [5] as well as result in recruitment of Sec23-Sec24. Typically defined by in vitro reconstitution assays, Sar1 in association with cargo and Sec23-24 has been termed the pre-budding complex. Within this pre-budding complex, Sec23 provides Sar1 GTPase-activating protein (GAP) activity and Sec24 provides a cargo-binding function [6]. The GAP activity of Sec23 is balanced at this pre-budding stage by the GEF activity of Sec12 [7]. The Sec13-Sec31 complex is recruited to form the outer layer of the coat and can further increase the GAP activity of Sec23 on Sar1 [8]. This results in rapid coat disassembly to form the mature vesicle; inherent to this mechanism is that coat disassembly is programmed into coat assembly.

The formation of vesicles at transitional ER presents a specific problem relating to the export of large secretory cargo from the ER; macromolecules measuring hundreds of nanometres must be accommodated within coated vesicles and these are typically described as carriers of 60$80 \mathrm{~nm}$ in diameter; such issues are not so apparent for COPI-mediated pathways, and much unusually large endocytic cargo is internalised via clathrin-independent mechanisms or following only partial assembly of a clathrin lattice $[9,10]$. It is important to note that the classical image of $60-80 \mathrm{~nm}$ coated vesicles arises largely from in vitro reconstitution assays performed in the presence of non-hydrolysable analogues of GTP (for example, [11]). The very presence of coated vesicles in 
mammalian cells remains controversial; while they are detectable [12], it is likely that only very few are present at any one time due to their rapid uncoating.

Premature disassembly of the coat is prevented by ongoing guanine nucleotide exchange on Sar1 catalysed by the ER-localised transmembrane protein, Sec12 $[7,13]$. Sec12 is excluded from the vesicle itself, which means that, once independent of the ER, the coat is stabilised only by coat-cargo interactions [13]; GTP hydrolysis therefore ultimately leads to coat disassembly. The presence of Sec12 in the ER membrane would maintain an active pool of Sar1-GTP at the bud neck [7], and from such in vitro reconstitution experiments (elegantly described in [14]), one can infer that nascent budding structures will contain more Sar1-GTP at the neck of a budding vesicle than within the core of the coat, which is likely to have key implications for vesicle fission $[5,15]$. Further factors are likely to be essential; for example, recruitment of Sar1 to mammalian membranes is ATPdependent [16]. In all cases, the COPII complex plays an integral role in the selection of cargo, and the features described above have key implications for the generation of both small and large transport carriers. Notably, intimate control of coating and uncoating is required to facilitate the incorporation of atypically large cargo.

\section{Major recent advances \\ Cranio-lenticulo-sutural dysplasia and chylomicron retention disease}

Recent work has shown that highly efficient coupling of Sec23-Sec24 to Sec13-Sec31 is required for export of collagen [17-19]. A mutation (F382L) in one of the two Sec23 genes in humans, sec23a, causes cranio-lenticulosutural dysplasia (CLSD) [20], a disorder characterised by craniofacial development defects. Mutation of $\sec 23 a$ in zebrafish also causes craniofacial development defects [18]. In these crusher mutant fish, chondrocytes contain extended ER compartments containing accumulated extracellular matrix proteins, notably collagen II, the major component of cartilage extracellular matrix, which is normally packaged as $300 \mathrm{~nm}$ procollagen bundles that exit the ER [18]. Accumulation of intracellular collagen is also seen in cells from CLSD patients. Recent data from the labs of Schekman, Orci, and Goldberg provide a detailed mechanistic explanation for the defects in COPII function seen in CLSD and crusher mutant zebrafish $[17,21]$. Elegant biochemical and structural data from the Goldberg lab show that the F382 residue sits on the surface of Sec23A where Sec31 binds as an extended polypeptide [21]; this region of Sec31 also contacts Sar1. Consistent with this failure to efficiently couple the inner and outer layers of the COPII coat in sec23a mutants, the gross phenotypes of collagen accumulation and craniofacial development defects are recapitulated by morpholino suppression in zebrafish of Sec13 [19]. Sec13 suppression in human fibroblasts drastically inhibits secretion of procollagen, but not the transport of small cargo. This suggests that the efficient and concerted assembly of the full coat is essential for transport of large cargoes [19].

Intriguingly, the two Sar1 proteins are expressed by many cell types. Sar1A and Sar1B have differing affinities for the Sec13-Sec31 complex [17], and Sar1A but not Sar1B was shown to partially rescue the F382L-Sec23A phenotype present in CLSD patients [17]. A prediction from the crystal structure that contains elements of Sar1, Sec23Sec24, and Sec31 [21] is that Sar1 and Sec31 interact at residues that differ between the two Sar1 isoforms. As highlighted previously [14], the structure of cages formed with Sec13-31 alone shows a structure composed of squares and triangles [22] rather than the hexagons and pentagons that are seen in the clathrin coat. This, coupled with relatively simple inter-subunit contacts, would make Sec13-Sec31 inherently able to accommodate a wide range of cargo sizes $[14,22]$.

Another human disorder, chylomicron retention disease (CMRD), is caused by mutations in sara2, which encodes Sar1B; loss of Sar1B causes severe fat malabsorption and retention of chylomicron-like particles in membranebound compartments [23]. Chylomicrons are large lipoprotein particles (hundreds of nanometres in diameter) that, like collagen, require the formation of large carriers for export from the ER. This supports the theory that Sar1B could be required selectively for transport of chylomicrons due to its weaker affinity for Sec13-Sec31 and thereby facilitate the formation of more flexible COPII cages [24]. However, as stated above, Sar1A can rescue the budding defect caused by the F382L mutation of Sec23A [17], at least in vitro, so Sar1B cannot be required specifically for large cargo export. While the tissue-specific defects seen in CLSD and CMRD could result from differences in the expression profile of Sar1 isoforms, it remains possible that additional cargospecific factors are involved.

\section{A role for specific cargo receptors}

Many cargo receptors have now been identified for a variety of processes, including ER-Golgi intermediate compartment (ERGIC)-53, Erv29p/Surf-4, and Emp24p [25-28]. While these transmembrane cargo adaptors can also engage the COPII complex, no concrete data exist to suggest that these play a major role in modulating the kinetics of COPII vesicle formation to facilitate largecarrier formation. This might reflect the fact that these proteins mediate the export of small freely diffusible 
cargoes. Recently, TANGO1 was characterised as a cargo receptor that could fulfil such a role for at least one macromolecular cargo [29]. TANGO1 was identified in a screen for regulators of transport and organisation of the ER/Golgi interface [30] and has been shown to be a collagen VII-binding protein that also links to the Sec23Sec24 layer of the COPII coat [29]. Suppression of TANGO1 blocks the secretion of collagen VII from cells but not that of collagen I, showing that it is not a general collagen receptor within the ER. It also differs from other known cargo receptors in that it localises to ERESs at a steady state [29]. TANGO1 is expressed ubiquitously and yet collagen VII is more restricted in its expression pattern [31]. This suggests that TANGO1 could act as a transport adaptor for other cargoes or play other roles relating to COPII function in other tissues.

TANGO1 could also play a role in the global organisation of ERES [29], but it remains unclear whether this relates to a direct effect on COPII dynamics or results from an accumulation of collagen within the ER following TANGO1 suppression. This latter possibility [29] is consistent with data showing that Sec13 suppression also leads to an accumulation of collagen within the ER and perturbation of ERES organisation [19]. However, this effect on COPII organisation was also seen in HeLa cells, which secrete very little collagen [19]. The role of TANGO1 is further complicated by the presence of an alternative truncated form of the protein (called TANGO or melanoma inhibitory activity family, member 3 [MIA3]) that is secreted [32] and modulates cell adhesion [33]. Expression of TANGO/MIA3 is reported to be downregulated in colon and hepatocellular carcinomas [34], which could also relate to a defect in epithelial organisation resulting from a loss of collagen VII secretion [35].

\section{Future directions}

Control of coat formation is clearly a key aspect to ensure incorporation of large cargo; the other key aspect, of course, is vesicle uncoating. The role of cargo receptors in the export of these macromolecular cargoes remains unclear. One can hypothesise that the accumulation of GTP-loaded Sar1 at bud sites could direct both membrane deformation and, through concerted local GTP hydrolysis, fission $[5,15]$ (Figure 1). This could require selective stabilisation of Sar1-GTP at the neck of nascent buds by Sec16 [36] or TANGO1 [29] (Figure 1). This would be consistent with these proteins being excluded from budding vesicles as has been shown for TANGO1 [29]. The fission step, in particular, remains ill defined here. A key future direction is to study how these factors, as well as the isoforms of core COPII subunits (such as Sar1A versus Sar1B), affect the kinetics and thermodynamics of COPII assembly.
Figure I. Possible mechanisms to couple coat protein II (COPII) assembly to the export of collagen

A

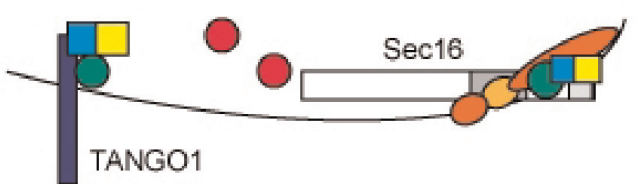

B
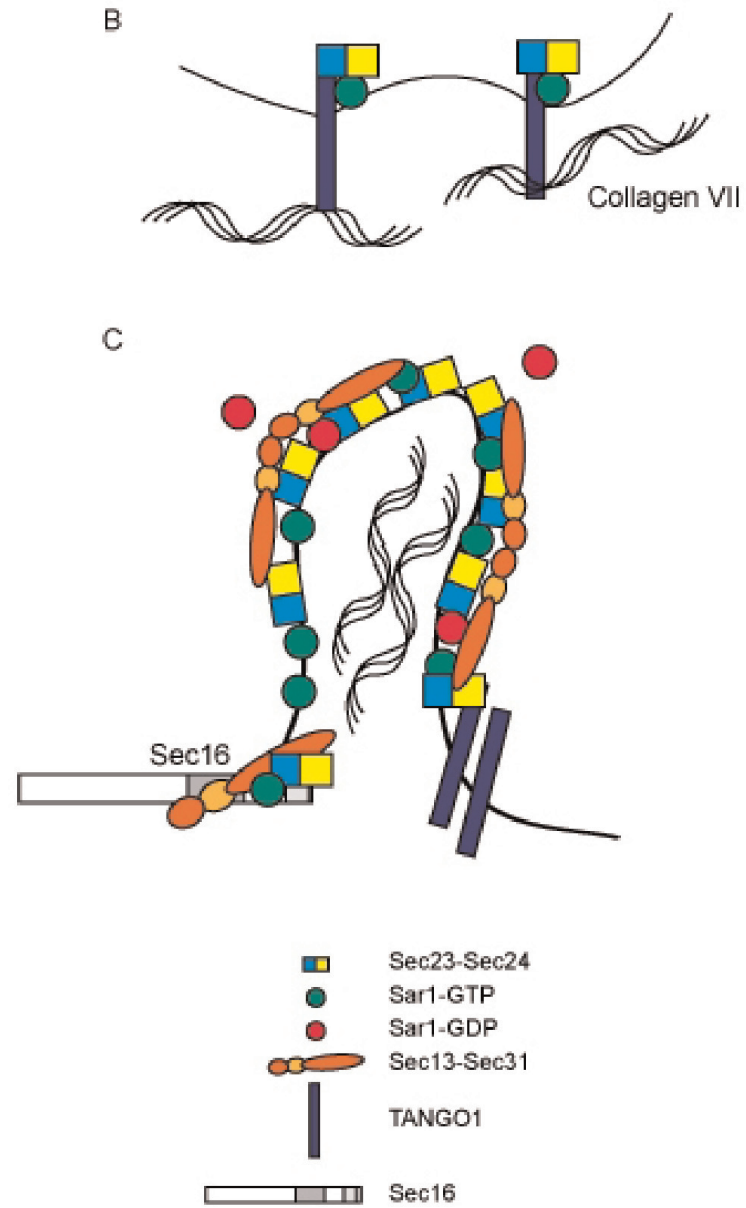

(A) TANGOI and Secl6 both have the capacity to interact with COPII components to increase the local pool of 'active' COPII. Such clustering of Sarl-GTP could induce membrane curvature to initiate bud formation. (B) TANGO can also interact with collagen VII. However, it is not clear whether TANGOI can engage both collagen VII and Sec23-Sec24 simultaneously. (C) Together, these properties provide a potential mechanism to couple the incorporation of cargo with the organisation of COPII assembly. Whether either Secl6 or TANGOI can modulate the kinetics of GTP hydrolysis by Sar I, or other aspects of COPII assembly, remains to be determined. Furthermore, it is not known whether the engagement of cargo by TANGOI has any impact on COPII dynamics. Assembly of Secl3-Sec3I is also required for efficient export of collagen I, indicating that coordinated assembly of COPII is central to the export of collagen from the endoplasmic reticulum. GDP, guanine diphosphate; GTP, guanine triphosphate. 
For larger carriers, the question remains open as to whether completion of coat formation is absolutely required [37] or whether partial coats analogous to those seen during endocytic entry of vesicular stomatitis virus can form [9]. One could envisage that the loss of assembled coat at regions of buds more distal to the neck could occur before the completion of scission. Data support the idea that the amount of Sar1-GTP is different within the 'older' part of the coat compared with that at the bud neck, the 'newer' part $[7,14,38]$. This would, despite transient stabilisation by interaction with cargo [13], ultimately result in the loss of Sec23-Sec24 and Sec13-Sec31. This could explain the presence in EM sections of apparent export carriers that are not coated with COPII in the vicinity of bona fide COPII-coated export sites [37]. Further immunolabelling and EM tomography of relevant cell types in an unperturbed state, or even tissue sections, would likely resolve this. Complete cage assembly could be necessary to encapsulate macromolecular cargo and this would be consistent with a selective defect in collagen secretion following knockdown of Sec13-Sec31 [19]. The use of metazoan model systems such as zebrafish has the potential to provide key physiologically relevant insights here.

In summary, recent data indicate that tight control over coated vesicle formation is required to direct the incorporation of large cargo into nascent buds; such control necessarily extends to the process of uncoating. Directed coat assembly, coupled with spatial restriction of GTPase activity and the activity of cargo receptors such as TANGO1, provides a concerted mechanism to ensure efficient incorporation of these larger cargoes into cells. From these data, it is becoming clear that the fine-tuning of the kinetics and thermodynamics of COPII assembly is essential for the export of many atypical secretory cargo molecules from the ER, and this has clear implications for human development, health, and disease.

\section{Abbreviations}

CLSD, cranio-lenticulo-sutural dysplasia; CMRD, chylomicron retention disease; COPII, coat protein II; EM, electron microscopy; ER, endoplasmic reticulum; ERES, endoplasmic reticulum exit site; ERGIC, ER-Golgi intermediate compartment; GAP, GTPase-activating protein; GEF, guanine nucleotide exchange factor; GDP, guanine diphosphate; GTP, guanine triphosphate; MIA3, melanoma inhibitory activity family, member 3 .

\section{Competing interests}

The authors declare that they have no competing interests.

\section{Acknowledgments}

The authors thank members of the lab of DJS for critical reading of the manuscript and the reviewers for their very helpful comments. Work in the lab of DJS is generously funded by the Medical Research Council and the Biotechnology and Biological Sciences Research Council.

\section{References}

I. Bonifacino JS, Glick BS: The mechanisms of vesicle budding and fusion. Cell 2004, I I6: I53-66.

2. Barlowe C, Orci L, Yeung T, Hosobuchi M, Hamamoto S, Salama N, Rexach MF, Ravazzola M, Amherdt M, Schekman R: COPII: a membrane coat formed by Sec proteins that drive vesicle budding from the endoplasmic reticulum. Cell 1994, 77:895-907.

3. Rossanese OW, Soderholm J, Bevis BJ, Sears IB, O'Connor J, Williamson EK, Glick BS: Golgi structure correlates with transitional endoplasmic reticulum organization in Pichia pastoris and Saccharomyces cerevisiae. J Cell Biol 1999, I45:69-8I.

4. Hughes $\mathrm{H}$, Stephens DJ: Assembly, organization, and function of the COPII coat. Histochem Cell Biol 2008, I 29:I29-5I.

5. Lee MC, Orci L, Hamamoto S, Futai E, Ravazzola M, Schekman R: Sarlp $\mathbf{N}$-terminal helix initiates membrane curvature and completes the fission of a COPII vesicle. Cell 2005, I 22:605-I 7.

FI000 Factor 9.6 Exceptional

Evaluated by Anne Spang 16 Sept 2005, David Stephens 19 Jan 2006

6. Miller EA, Beilharz TH, Malkus PN, Lee MC, Hamamoto S, Orci L, Schekman R: Multiple cargo binding sites on the COPII subunit Sec24p ensure capture of diverse membrane proteins into transport vesicles. Cell 2003, I I 4:497-509.

FI000 Factor 10.3 Exceptional

Evaluated by Craig Roy 05 Sept 2003, Anne Spang 05 Sept 2003,

Tom Stevens 10 Sept 2003, Tom Rapoport II Sept 2003, David

Lambright 16 Sept 2003

7. Futai E, Hamamoto S, Orci L, Schekman R: GTP/GDP exchange by SecI2p enables COPII vesicle bud formation on synthetic liposomes. EMBO / 2004, 23:4|46-55.

8. Antonny B, Gounon P, Schekman R, Orci L: Self-assembly of minimal COPII cages. EMBO Rep 2003, 4:4I9-24.

FI000 Factor 6.0 Must Read

Evaluated by Rainer Duden 25 Apr 2003

9. Cureton DK, Massol RH, Saffarian S, Kirchhausen TL, Whelan SP: Vesicular stomatitis virus enters cells through vesicles incompletely coated with clathrin that depend upon actin for internalization. PLoS Pathog 2009, 5:el000394.

FI000 Factor 3.0 Recommended

Evaluated by Marc Lecuit 28 May 2009

10. Doherty GJ, McMahon HT: Mechanisms of endocytosis. Annu Rev Biochem 2009, 78:857-902.

II. Matsuoka K, Orci L, Amherdt M, Bednarek SY, Hamamoto S, Schekman R, Yeung T: COPII-coated vesicle formation reconstituted with purified coat proteins and chemically defined liposomes. Cell 1998, 93:263-75.

12. Zeuschner D, Geerts WJ, van Donselaar E, Humbel BM, Slot JW, Koster AJ, Klumperman J: Immuno-electron tomography of ER exit sites reveals the existence of free COPII-coated transport carriers. Nat Cell Biol 2006, 8:377-83.

FI000 Factor 8.2 Must Read

Evaluated by David Stephens 20 Mar 2006, Jesse Hay 03 Oct 2007, Ariel Blocker 12 Apr 2006

13. Sato K, Nakano A: Dissection of COPII subunit-cargo assembly and disassembly kinetics during Sarlp-GTP hydrolysis. Nat Struct Mol Biol 2005, I 2:167-74.

FI000 Factor 6.0 Must Read

Evaluated by David Stephens 29 Apr 2005 
14. Antonny B: Membrane deformation by protein coats. Curr Opin Cell Biol 2006, I 8:386-94.

I5. Bielli A, Haney CJ, Gabreski G, Watkins SC, Bannykh SI, Aridor M: Regulation of Sarl NH2 terminus by GTP binding and hydrolysis promotes membrane deformation to control COPII vesicle fission. J Cell Biol 2005, I 7 I:919-24.

16. Aridor M, Balch WE: Kinase signaling initiates coat complex II (COPII) recruitment and export from the mammalian endoplasmic reticulum. J Biol Chem 2000, 275:35673-6.

17. Fromme JC, Ravazzola M, Hamamoto S, Al-Balwi M, Eyaid W, Boyadjiev SA, Cosson P, Schekman R, Orci L: The genetic basis of a craniofacial disease provides insight into COPII coat assembly. Dev Cell 2007, I3:623-34.

FI000 Factor 6.5 Must Read

Evaluated by Charles Barlowe II Dec 2007, David Stephens I3 Dec 2007, Jane Hewitt 04 Mar 2008

18. Lang MR, Lapierre LA, Frotscher M, Goldenring JR, Knapik EW: Secretory COPII coat component Sec23a is essential for craniofacial chondrocyte maturation. Nat Genet 2006, 38: I I 98-203.

FI000 Factor 3.0 Recommended

Evaluated by David Stephens 03 Oct 2006

19. Townley AK, Feng Y, Schmidt K, Carter DA, Porter R, Verkade P, Stephens DJ: Efficient coupling of Sec23-Sec24 to Sec I3-Sec3 I drives COPII-dependent collagen secretion and is essential for normal craniofacial development. J Cell Sci 2008, I 2 I:3025-34.

20. Boyadjiev SA, Fromme JC, Ben J, Chong SS, Nauta C, Hur DJ, Zhang G, Hamamoto S, Schekman R, Ravazzola M, Orci L, Eyaid W: Cranio-lenticulo-sutural dysplasia is caused by a SEC23A mutation leading to abnormal endoplasmic-reticulum-toGolgi trafficking. Nat Genet 2006, 38: I192-7.

FI000 Factor 6.0 Must Read

Evaluated by David Stephens 03 Oct 2006

21. Bi X, Mancias JD, Goldberg J: Insights into COPII coat nucleation from the structure of Sec23*Sar I complexed with the active fragment of Sec3 I. Dev Cell 2007, I3:635-45.

FI000 Factor 3.0 Recommended

Evaluated by Charles Barlowe II Dec 2007

22. Stagg SM, Gurkan C, Fowler DM, LaPointe P, Foss TR, Potter CS, Carragher B, Balch WE: Structure of the Sec I3/3 I COPII coat cage. Nature 2006, 439:234-8.

FI000 Factor 6.6 Must Read

Evaluated by Charles Barlowe 18 Jan 2006, David Stephens 19 Jan 2006, Tony Crowther 20 Jan 2006

23. Jones B, Jones EL, Bonney SA, Patel HN, Mensenkamp AR, Eichenbaum-Voline S, Rudling M, Myrdal U, Annesi G, Naik S, Meadows N, Quattrone A, Islam SA, Naoumova RP, Angelin B, Infante R, Levy E, Roy CC, Freemont PS, Scott J, Shoulders CC: Mutations in a Sar I GTPase of COPII vesicles are associated with lipid absorption disorders. Nat Genet 2003, 34:29-3I.

FI000 Factor 3.0 Recommended

Evaluated by Rainer Duden 25 Apr 2003

24. Stagg SM, LaPointe P, Razvi A, Gurkan C, Potter CS, Carragher B, Balch WE: Structural basis for cargo regulation of COPII coat assembly. Cell 2008, 134:474-84.

FI000 Factor 6.0 Must Read

Evaluated by David Stephens 20 Aug 2008

25. Nichols WC, Seligsohn U, Zivelin A, Terry VH, Hertel CE, Wheatley MA, Moussalli MJ, Hauri HP, Ciavarella N, Kaufman RJ, Ginsburg D: Mutations in the ER-Golgi intermediate compartment protein ERGIC-53 cause combined deficiency of coagulation factors V and VIII. Cell 1998, 93:6I-70.
26. Appenzeller C, Andersson H, Kappeler F, Hauri HP: The lectin ERGIC-53 is a cargo transport receptor for glycoproteins. Nat Cell Biol 1999, I:330-4

27. Belden WJ, Barlowe C: Role of Erv29p in collecting soluble secretory proteins into ER-derived transport vesicles. Science 200|, 294: |528-3|.

FI000 Factor 6.5 Must Read

Evaluated by Anne Spang 2I Nov 200I, Akihiko Nakano 20 Dec 2001, Susan Henry 22 Jan 2002

28. Muniz M, Nuoffer C, Hauri HP, Riezman H: The Emp24 complex recruits a specific cargo molecule into endoplasmic reticulum-derived vesicles. J Cell Biol 2000, I48:925-30.

29. Saito K, Chen M, Bard F, Chen S, Zhou H, Woodley D, Polischuk R, Schekman R, Malhotra V: TANGOI facilitates cargo loading at endoplasmic reticulum exit sites. Cell 2009, I36:89|-902.

FI000 Factor 3.0 Recommended

Evaluated by David Stephens 19 Mar 2009

30. Bard F, Casano L, Mallabiabarrena A, Wallace E, Saito K, Kitayama H, Guizzunti G, Hu Y, Wendler F, Dasgupta R, Perrimon N, Malhotra V: Functional genomics reveals genes involved in protein secretion and Golgi organization. Nature 2006, 439:604-7.

FI000 Factor 3.2 Recommended

Evaluated by Jane Hewitt 14 Feb 2006, Jesse Hay 03 Oct 2007

3I. Wetzels RH, Robben HC, Leigh IM, Schaafsma HE, Vooijs GP, Ramaekers FC: Distribution patterns of type VII collagen in normal and malignant human tissues. Am J Pathol 1991, | 39:45|-9.

32. Bosserhoff AK, Moser M, Buettner R: Characterization and expression pattern of the novel MIA homolog TANGO. Gene Expr Patterns 2004, 4:473-9.

33. Arndt S, Melle C, Mondal K, Klein G, von Eggeling F, Bosserhoff AK: Interactions of TANGO and leukocyte integrin CD I I c/CD I 8 regulate the migration of human monocytes. J Leukoc Biol 2007, 82: $1466-72$.

34. Arndt S, Bosserhoff AK: Reduced expression of TANGO in colon and hepatocellular carcinomas. Oncol Rep 2007, | 8:885-9|.

35. Saaf AM, Halbleib JM, Chen X, Yuen ST, Leung SY, Nelson WJ, Brown PO: Parallels between global transcriptional programs of polarizing Caco-2 intestinal epithelial cells in vitro and gene expression programs in normal colon and colon cancer. Mol Biol Cell 2007, I 8:4245-60.

36. Ivan V, de Voer G, Xanthakis D, Spoorendonk KM, Kondylis V, Rabouille C: Drosophila Secl 6 mediates the biogenesis of tER sites upstream of Sar I through an arginine-rich motif. Mol Biol Cell 2008, 19:4352-65.

FI000 Factor 3.0 Recommended

Evaluated by David Stephens 20 Aug 2008

37. Mironov AA, Beznoussenko GV, Trucco A, Lupetti P, Smith JD, Geerts WJ, Koster AJ, Burger KN, Martone ME, Deerinck T], Ellisman MH, Luini A: ER-to-Golgi carriers arise through direct en bloc protrusion and multistage maturation of specialized ER exit domains. Dev Cell 2003, 5:583-94.

FI000 Factor 8.0 Must Read

Evaluated by Ron Prywes 28 Nov 2003, Sebastian Springer 02 Dec 2003

38. Antonny B, Madden D, Hamamoto S, Orci L, Schekman R: Dynamics of the COPII coat with GTP and stable analogues. Nat Cell Biol 200I, 3:53I-7.

FI000 Factor 6.0 Must Read

Evaluated by Catherine Jackson 28 Sept 2001 